syndrome. Relatively few investigators had stressed the importance of mesencephalic and rhrombencephalic involvement in association with cerebral cortical dysgenesis until the work of Sarnat, colleagues and others in the 1990s. A classification of cerebral malformations proposed by Sarnat et al in 2004 also stressed the inclusion of thalamic, brainstem and cerebellar malformations in association with lissencephaly and holoprosencephaly. In an editorial, Dr Sarnat reviews the progress of our understanding of the genetic programming of neural tube development and the need for research on a genetic mechanism for the association of forebrain and hindbrain malformations in the lissencephalies. (Sarnat HB. Cortical malformations. Looking behind the cortex. Neurology 2009;72:394-395).

\title{
SURGICAL OUTCOME IN FOCAL CORTICAL DYSPLASIA
}

The predictors of surgical outcome and relevance of pathological severity were determined in 166 consecutive patients with intractable epilepsy and focal cortical dysplasias treated surgically at Konkuk University Medical Center, and National University Hospital, Seoul, Korea. Poor surgical outcome was associated with incomplete resection of epileptogenic area, mild pathologic features, and secondary tonic-clonic seizures. Patients with severe pathologic features had MRI abnormalities. MRI findings, EEG, PET and ictal SPECT were not associated with surgical outcomes. (Kim DW, Lee SK, Chu K, et al. Predictors of surgical outcome and pathologic considerations in focal cortical dysplasia. Neurology Jan 20, 2009;72:211-216). (Respond and Reprints: Dr Sang Kun Lee, Department of Neurology, Seoul National University Hospital 28, Chongno Ku, Seoul ,110-744, Korea. E-mail: sangunlee@dreamwiz.com).

COMMENT. Patients with focal cortical dysplasia and intractable epilepsy are at risk of a poor surgical outcome, when associated with incomplete resection, mild pathologic features, or secondary tonic clonic seizures. Incomplete resection of focal cortical dysplasia was the main predictor of poor postsurgical outcome in 149 pediatric patients operated at the Miami Children's Hospital (Krsek P et al. Neurology 2009;72:217-223).

In practice, a negative MRI does not exclude a subtle cortical dysplasia that may underly refractory seizures. Newer imaging techniques may uncover small dysplasias amenable to treatment in specialized epilepsy and surgical centers. (Mathern GW. Neurology 2009;72:206-207).

\section{VASCULAR DISORDERS}

\section{INTRACRANIAL ARTERIOPATHY AND ISCHEMIC STROKE}

Repeated vascular imaging findings and clinical charts of 79 children with anterior circulation arterial ischemic stroke (AIS) and unilateral intracranial arteriopathy of the internal carotid bifurcation were studied at the University Medical Center, Utrecht, The Netherlands, and other centers in France, UK, and Canada. The characteristics of $5(6 \%)$ patients with progressive and 74 (94\%) with transient cerebral arteriopathy (TCA) were compared after a median follow-up of 1.4 years. Most infarcts were localized in the basal ganglia. Follow-up vascular imaging showed complete normalization in $23 \%$ of TCA 
patients; $77 \%$ had residual arterial abnormalities, with improvement in $45 \%$ and stabilization in $32 \%$. Before the arteriopathy stabilized or improved, transient worsening occurred in 14 $(19 \%)$ of TCA patients; $13(18 \%)$ had a recurrent stroke or TIA. Stroke was preceded by chickenpox in $44 \%$ of TCA patients and in none of those with progressive arteriopathies. Neurological outcome was good in $30(41 \%)$ of the TCA patients and in none of the 5 with progressive arteriopathy. Progressive arteriopathy was associated with arterial occlusion, moyamoya disease and anterior cerebral artery involvement, and with stroke recurrence. (Braun KPJ, Bulder MMM, Chabrier S, et al. The course and outcome of unilateral intracranial arteriopathy in 79 children with ischemic stroke. Brain Feb 2009;132:544-557). (Respond: KPJ Braun MD, PhD, Department of Child Neurology, Rudolf Magnus Institute of Neuroscience, Wilhelmina Children's Hospital, University Medical Center Utrecht, PO Box 85500, 3508 GA Utrecht, the Netherlands. E-mail: k.braun@umcutrecht.nl).

COMMENT. In contrast to adults with stroke, the majority of childhood arterial ischemic strokes are caused by non-atherosclerotic arterial disease. Transient cerebral arteriopathy is a common cause and is characterized by infarction in the lateral lenticulostriate territory. The majority of patients in the above study had unilateral transient cerebral arteriopathy, and stroke was preceded by chickenpox (post-varicella arteriopathy) in $44 \%$. Evidence supports a post-infectious inflammatory mechanism underlying transient cerebral arteriopathy.

\section{CLASSIFICATION OF PERINATAL ISCHEMIC STROKE}

Advances in classification, causes, treatment and outcome of perinatal ischemic stroke are reviewed by researchers at Alberta Children's Hospital, Calgary; and the Hospital for Sick Children, Toronto, Canada. Four perinatal stroke syndromes are described in order of decreasing incidence: 1) neonatal arterial ischemic stroke (accounts for two-thirds); 2) neonatal cerebral sinovenous thrombosis; 3) presumed perinatal ischemic stroke; and 4) periventricular venous infarction. MRI of the head and neck is the investigation of choice in perinatal stroke. Diffusion-weighted MRI provides information on timing for acute, focal infarction. MR angiography defines arterial occlusion or arteriopathy. MR venography defines location and extent of venous thrombosis, and diffusion weighted MRI confirms or excludes venous edema or infarction.

Maternal, prenatal, and placental risk factors include chorioamnionitis, prolonged rupture of membranes, preeclampsia, placental thrombi and intrauterine growth retardation. Intrapartum factors include neonatal encephalopathy with asphyxia that may co-occur with perinatal stroke. Prothrombotic and hematological factors occur in $20-68 \%$ of neonates with ischemic stroke. Cardiac evaluation is recommended in all cases of perinatal stroke, and complex congenital heart disease predisposes neonates to cerebral thromboembolism, especially during diagnostic and surgical procedures. Infection and inflammation are common in perinatal stroke, and the association with bacterial meningitis and chorioamnionitis requires further study.

Treatment involves maternal prevention approaches (counseling regarding smoking, weight control), rescue at risk brain tissue (anticoagulation, maintain normal blood sugar, temperature, oxygenation, blood pressure), and optimize outcome (study of hypothermia, treatment of seizures). Congenital hemiplegia and epilepsy are the most common 